\title{
Marker assisted selection with optimised contributions of the candidates to selection
}

\author{
Beatriz Villanueva ${ }^{\mathrm{a} *}$, Ricardo Pong-Wong ${ }^{\mathrm{b}}$, \\ John A. Woolliams ${ }^{b}$ \\ a Scottish Agricultural College, West Mains Road, \\ Edinburgh, EH9 3JG, Scotland, UK \\ ${ }^{\mathrm{b}}$ Roslin Institute (Edinburgh), Roslin, Midlothian, EH25 9PS, Scotland, UK
}

(Received 13 November 2001; accepted 2 August 2002)

\begin{abstract}
The benefits of marker assisted selection (MAS) are evaluated under realistic assumptions in schemes where the genetic contributions of the candidates to selection are optimised for maximising the rate of genetic progress while restricting the accumulation of inbreeding. MAS schemes were compared with schemes where selection is directly on the QTL (GAS or gene assisted selection) and with schemes where genotype information is not considered (PHE or phenotypic selection). A methodology for including prior information on the QTL effect in the genetic evaluation is presented and the benefits from MAS were investigated when prior information was used. The optimisation of the genetic contributions has a great impact on genetic response but the use of markers leads to only moderate extra short-term gains. Optimised PHE did as well as standard truncation GAS (i.e. with fixed contributions) in the short-term and better in the long-term. The maximum accumulated benefit from MAS over PHE was, at the most, half of the maximum benefit achieved from GAS, even with very low recombination rates between the markers and the QTL. However, the use of prior information about the QTL effects can substantially increase genetic gain, and, when the accuracy of the priors is high enough, the responses from MAS are practically as high as those obtained with direct selection on the QTL.
\end{abstract}

marker assisted selection / gene assisted selection / optimised selection / BLUP selection / restricted inbreeding

\section{INTRODUCTION}

The rapid advances in molecular genetic technologies in the last decades have greatly increased the chances of identifying quantitative trait loci (QTL) or markers linked to such loci in livestock species. A considerable number of markers linked to economically important traits are now available e.g. [1,5,6, $16]$ and this is likely to increase in the next few years. Markers linked to QTLs

* Correspondence and reprints

E-mail: b.villanueva@ed.sac.ac.uk 
can be used as an aid in selection decisions to increase the accuracy of selection and thus genetic gain.

Statistical methods have been developed for using marker information in BLUP (best linear unbiased prediction) genetic evaluations $[4,9,12,15,29,33]$. BLUP methodology allows simultaneous estimation of both the QTL and the polygenic effects. The QTL effect is accounted for in the mixed model as an extra random effect with covariance structure proportional to the IBD (identityby-descent) matrix at the QTL position given the linked markers. Thus, the evaluation is not restricted to a given type of pedigree structure.

Studies investigating the value of marker assisted selection (MAS) for increasing genetic response in outbred populations have found extra (although variable) gains e.g. [20,22,26,27], particularly for sex-limited and lowly heritable traits. These studies have compared MAS and conventional schemes based on rates of genetic gain obtained with standard truncation selection where the number of parents selected and their contributions are fixed. Thus with standard truncation selection, both types of schemes could lead not only to different rates of genetic gain but also to different rates of inbreeding.

The use of selection algorithms that optimise the contributions of the selection candidates for obtaining maximum genetic gains while restricting the rate of inbreeding give higher gains than truncation selection and allow to compare schemes at the same rate of inbreeding [13,19]. Studies of the benefits of these techniques $[2,3,19]$ suggest approximately $20 \%$ improvements in the rates of gain and higher over conventional truncation BLUP.

These optimisation procedures have been proven to work well when selection is directly on a major gene that is segregating in addition to the polygenes. Villanueva et al. [31] showed that optimised selection gave higher gains than truncation selection and was able to constrain the increase in inbreeding to the desired value under this type of a mixed inheritance model. Also, they showed that the conflict between long- and short-term responses from explicit use of the known gene $[10,11,17,24]$ can be resolved in schemes with constrained inbreeding, and where the basis of evaluation is BLUP, but only under some scenarios (e.g. when the gene had a large effect).

Previous research on the optimisation of schemes using information on a QTL has assumed that all individuals have a known genotype for the QTL and that its effect is known without error [30,31]. This assumption may not often hold in practise and markers, rather than known genes, are more likely to be used. On the contrary, previous studies on MAS have not considered the rate of inbreeding. In this study we extended the optimisation method for maximising gain while restricting the rate of inbreeding, to include selection on genetic markers rather than on the QTL itself. The optimisation algorithm uses BLUP breeding values obtained by using the methodology of Fernando and Grossman [9] and pedigree data. Expected genetic gains from GAS and 
MAS schemes were compared. Also, in order to investigate the reasons for differences in response between GAS and MAS, the benefits obtained from MAS when independent prior information about the QTL effects was used in the genetic evaluation were evaluated. Rates of gain obtained from different schemes were compared at fixed rates of inbreeding.

\section{METHODS}

Three types of schemes were compared using Monte Carlo simulations: (1) phenotypic selection (PHE): selection ignoring information on the QTL or on the markers when estimating breeding values (EBVs); (2) gene assisted selection (GAS): selection using information on the QTL assuming that its effect is known and that all individuals have a known genotype for the QTL; and (3) marker assisted selection (MAS): selection using information on markers linked to the QTL (i.e. assuming that the effect and the genotypes for the QTL are unknown). BLUP genetic evaluation was used in the three types of schemes. Although the optimisation algorithm was used to evaluate the benefit from MAS over conventional selection (PHE), schemes under standard truncation selection were also run for comparison. With "optimised selection", the numbers of parents and their contributions were optimised each generation to maximise genetic gain while restricting the rate of inbreeding. With truncation selection, the number of parents and the family sizes were fixed across generations.

\subsection{Genetic model}

The trait under selection was genetically controlled by an infinite number of additive loci, each with an infinitesimal effect (polygenes) plus a single biallelic (alleles $\mathrm{A}_{1}$ and $\mathrm{A}_{2}$ ) locus (QTL). The total genetic value of the $i$ th individual was $g_{i}=v_{i}+u_{i}$, where $v_{i}$ is the genotypic value due to the QTL and $u_{i}$ is the polygenic effect. The QTL had an additive effect (a), defined as half the difference between the two homozygotes. Thus the genotypic value due to the QTL was $a, 0$ and $-a$ for individuals with the genotype $\mathrm{A}_{1} \mathrm{~A}_{1}, \mathrm{~A}_{1} \mathrm{~A}_{2}$ and $\mathrm{A}_{2} \mathrm{~A}_{2}$, respectively. The genetic variance explained by the QTL in the initial population was $\sigma_{v}^{2}=2 p(1-p) a^{2}$, where $p$ is the initial frequency of the favourable allele $A_{1}[8]$. In addition to the polygenes and the QTL affecting the trait, a set of polymorphic marker loci linked to the QTL were simulated. The markers were flanking the QTL and they did not have any effect on the selected trait. At least six alleles of equal frequencies were simulated for each marker. Most simulations were run with two flanking markers.

\subsection{Simulation of the population}

The base population $(t=0)$ was composed of $N$ individuals $(N / 2$ males and $N / 2$ females) with family structure. A number $g_{\text {random }}$ of prior generations 
$(t<0)$ of random selection were simulated to create this family structure. In most simulations, $g_{\text {random }}$ was set to one. The initial population was composed of $N$ unrelated individuals. Random selection of $N_{s o}$ males and $N_{d o}$ females was applied to generations $t<0$. Generation $1(t=1)$ was obtained from the mating of individuals selected at $t=0$. The number of selection candidates $(N)$ was kept constant across generations. In the initial population, the polygenic effect for each individual was obtained from a normal distribution with mean zero and variance $\sigma_{u}^{2}$. The alleles at the QTL and the markers were chosen at random with appropriate probabilities (i.e. those given by the initial allele frequencies). The markers, QTL and polygenes were in linkage phase equilibrium. The phenotypic value for an individual $i\left(y_{i}\right)$ was obtained by adding a normally distributed environmental component $\left(e_{i}\right)$ with mean zero and variance $\sigma_{e}^{2}$ to the total genetic value $\left(g_{i}\right)$.

In subsequent generations, the polygenic effect of the offspring was generated as the average of the polygenic effects of their parents plus a random Mendelian deviation. The latter was sampled from a normal distribution with mean zero and variance $\left(\sigma_{u}^{2} / 2\right)\left[1-\left(F_{s}+F_{d}\right) / 2\right]$, where $F_{s}$ and $F_{d}$ are the inbreeding coefficients of the sire and dam, respectively. Marker and QTL alleles were transmitted from parents to offspring in classical Mendelian fashion, allowing for recombination. The Haldane mapping function (e.g. [18]) was used to obtain the relationship between the distance between two loci and their recombination frequency. In MAS schemes, all individuals are assumed to be genotyped each generation for the marker loci (including $t<0$ ). In GAS schemes, all individuals were assumed to be genotyped for the QTL.

\subsection{Estimation of breeding values}

Gains obtained in schemes where genetic evaluation makes use of markers linked to the QTL were compared to those obtained in schemes where the QTL effect was assumed to be known and to those obtained in schemes that ignored all the genotype information on the QTL and the markers.

\subsubsection{Schemes ignoring genotype information (PHE)}

When the information on the QTL or on the markers was not used, genetic evaluations were entirely based on phenotypic and pedigree information. The total estimated breeding value for an individual $i\left(E B V_{i}\right)$ was obtained from standard BLUP using the total genetic additive variance $\left(\sigma_{v}^{2}+\sigma_{u}^{2}\right)$ of the base population and the phenotypic values uncorrected for the QTL effect.

\subsubsection{Schemes with direct selection on the QTL (GAS)}

In schemes selecting directly on the QTL, it was assumed that all individuals had a known genotype for the QTL and that its effect was known without error. 
In this case the estimated breeding value was:

$$
E B V_{i}=\hat{u}_{i}+w_{i}
$$

where $\hat{u}_{i}$ is the estimate of the polygenic breeding value and $w_{i}$ is the breeding value due to the QTL effect. The estimate $\hat{u}_{i}$ was obtained from standard BLUP using the polygenic variance $\left(\sigma_{u}^{2}\right)$ and the phenotypic values $\left(y_{i}\right)$ corrected for the QTL effect $\left(y_{i}^{\prime}=y_{i}-v_{i}\right)$. The breeding value for the QTL was $2(1-p) a$, $[(1-p)-p] a$ and $-2 p a$ for individuals with genotype $\mathrm{A}_{1} \mathrm{~A}_{1}, \mathrm{~A}_{1} \mathrm{~A}_{2}$ and $\mathrm{A}_{2} \mathrm{~A}_{2}$, respectively [8]. The frequency $p$ was updated each generation to obtain $w_{i}$.

\subsubsection{Schemes with selection on the markers (MAS)}

The estimation of breeding values when using information from markers linked to the QTL was carried out following the methodology of Fernando and Grossman [9]. The model used was:

$$
\mathbf{y}=\mathbf{X b}+\mathbf{Z u}+\mathbf{W} \mathbf{v}+\mathbf{e}
$$

where $\mathbf{y}$ is the vector of phenotypic values, $\mathbf{X}, \mathbf{Z}$ and $\mathbf{W}$ are known incidence matrices relating the observations to the fixed effects (b), the polygenic effects (u) and the QTL effects (v), respectively and $\mathbf{e}$ is the vector of residuals. Here, b only includes the population mean. The vector $\mathbf{v}$ contains two QTL effects for each individual $i$, one for the paternal allele and another one for the maternal allele ( $v_{i}^{p}$ and $v_{i}^{m}$, respectively).

Fernando and Grossman [9] showed that, assuming that the variances in the base population are known, both the polygenic and the QTL effects can be estimated using BLUP. The mixed model equations (MME) including the QTL effects are:

$$
\left(\begin{array}{ccc}
\mathbf{X}^{\prime} \mathbf{X} & \mathbf{X}^{\prime} \mathbf{Z} & \mathbf{X}^{\prime} \mathbf{W} \\
\mathbf{Z}^{\prime} \mathbf{X} & \mathbf{Z}^{\prime} \mathbf{Z}+\gamma_{1} \mathbf{A}^{-1} & \mathbf{Z}^{\prime} \mathbf{W} \\
\mathbf{W}^{\prime} \mathbf{X} & \mathbf{W}^{\prime} \mathbf{Z} & \mathbf{W}^{\prime} \mathbf{W}+\gamma_{2} \mathbf{G}^{-1}
\end{array}\right)\left(\begin{array}{c}
\hat{\mathbf{b}} \\
\hat{\mathbf{u}} \\
\hat{\mathbf{v}}
\end{array}\right)=\left(\begin{array}{c}
\mathbf{X}^{\prime} \mathbf{y} \\
\mathbf{Z}^{\prime} \mathbf{y} \\
\mathbf{W}^{\prime} \mathbf{y}
\end{array}\right)
$$

where $\mathbf{A}$ and $\mathbf{G}$ represent the covariance matrices between individuals for the polygenic and the QTL effects, respectively. Thus $\mathbf{A}$ and $\mathbf{G}$ are, respectively, the numerator relationship matrix and the gametic relationship matrix (or IBD matrix) at the QTL position given the genotypes of the linked marker loci with a known recombination rate with the QTL. The matrix $\mathbf{G}$ given the marker loci genotypes, was obtained using the deterministic approach of Pong-Wong et al. [23]. The inverse of the numerator relationship matrix, $\mathbf{A}$, was directly obtained using the rules of Henderson [14] and Quaas [25]. Finally, $\gamma_{1}$ and $\gamma_{2}$ are the variance ratios $\left(\sigma_{e}^{2} / \sigma_{u}^{2}\right.$ and $\sigma_{e}^{2} /\left[(0.5) \sigma_{v}^{2}\right]$, respectively) in the base population. 
The total estimated breeding value when MAS was applied was the sum of the estimates of the polygenic and the QTL effects obtained by solving the mixed model equations:

$$
E B V_{i}=\hat{u}_{i}+\hat{v}_{i}^{p}+\hat{v}_{i}^{m}
$$

\subsubsection{Inclusion of prior information on the QTL effect in the estimation of breeding values}

Information on the QTL effect obtained in, supposedly, independent QTL studies was included in the MME in order to investigate if this information could be used to increase the value of MAS.

Let us assume that, in addition to the marker genotypes and performance records, some candidates also have prior information about the QTL effect, which was obtained independently from previous QTL studies. For an individual $i, \hat{v}_{i}^{*}$ is a prior estimate of the combined additive effects of its two QTL alleles and this estimate has a certain accuracy $\left(\rho_{i}^{*}\right)$. This information can then be used in the genetic evaluation to increase the accuracy of the estimates of the QTL effects.

The prior information was included into the MAS evaluation by adding information of "phantom" offspring into the MME. Thus for an individual $i, n_{i}$ "phantom" half sib offspring were created, each having one phenotypic observation $\left(y_{o(i)}^{*}\right)$. The specific modifications carried out in the MME are detailed in Appendix A. The number of "phantom" offspring $\left(n_{i}\right)$ and their phenotypic value $\left(y_{o(i)}^{*}\right)$ are functions of $\hat{v}_{i}^{*}$ and $\rho_{i}^{*}$ as described in Appendix B. The marker genotypes of the "phantom" offspring were assumed to be noninformative (i.e. the offspring were not genotyped for the markers).

\subsection{Selection procedures}

The benefit of using markers was evaluated using a selection tool that optimises each generation for the contributions of the selection candidates. For purposes of comparison, the schemes under standard truncation selection (i.e. static schemes with fixed numbers of parents) were also simulated.

\subsubsection{Optimised selection}

With this type of selection, the numbers of individuals selected and their contributions are optimised for maximising genetic progress while restricting the rate of inbreeding to a specific value. The inbreeding rate considered here was computed from the pedigree based numerator relationship $\mathbf{A}$ matrix (i.e. it refers to the average inbreeding of the genome). The optimal solutions $\left(\mathbf{c}_{t}\right)$ were found by maximising the function described in Meuwissen [19]:

$$
H_{t}=\mathbf{c}_{t}^{\mathrm{T}} \mathbf{E B} \mathbf{V}_{t}-\lambda_{0}\left(\mathbf{c}_{t}^{\mathrm{T}} \mathbf{A}_{t} \mathbf{c}_{t}-C_{t}\right)-\left(\mathbf{c}_{t}^{\mathrm{T}} \mathbf{Q}-\frac{1}{2} \mathbf{1}^{\mathrm{T}}\right) \lambda
$$


where $\mathbf{c}_{t}$ is the vector of contributions to the next generation of the $N$ selection candidates available at generation $t, \mathbf{E B V}$ is the vector of their estimated breeding values (described before for the three types of schemes), $\mathbf{A}$ is the numerator relationship matrix of the candidates, $\mathbf{Q}$ is a known incidence matrix $N \times 2$ with ones for males and zeros for females in the first column and ones for females and zeros for males in the second column, $C$ is the constraint on the rate of inbreeding as described in Grundy et al. [13] $\left(C_{t}=2\left[1-(1-\Delta F)^{t}\right]\right.$, where $\Delta F$ is the desired rate of inbreeding), 1 is a vector of ones of order 2 and $\lambda_{0}$ and $\lambda$ (a vector of order 2) are Lagrangian multipliers.

The solutions obtained with this algorithm $\left(\mathbf{c}_{t}\right)$ are expressed as mating proportions which sum to a half for each sex. The optimal number of offspring (integer) for each parent was obtained from $\mathbf{c}_{t}$ as described in Grundy et al. [13]. Each parent was randomly allocated to different mates (among the selected individuals) to produce its offspring.

It should be noted that the optimisation applied here differs from that described by Dekkers and van Arendonk [7] where the purpose was to achieve the optimal emphasis given to the QTL relative to the polygenes across generations for maximising gain in truncation selection schemes. Dekkers and van Arendonk [7] considered infinite populations and therefore no accumulation of inbreeding.

\subsubsection{Truncation selection}

With standard truncation selection, a fixed number of individuals $\left(N_{s}\right.$ males and $N_{d}$ females) with the highest estimated breeding values are selected to be parents of the next generation. Matings were hierarchical with each sire being mated at random to $N_{d} / N_{s}$ dams and each dam being mated to a single sire. Each dam produced the same number of offspring of each sex (i.e. $N / 2 N_{d}$ males and $N / 2 N_{d}$ females).

\subsection{Parameters studied}

In the scheme used as a reference (basic scheme), a single extra generation was generated to create a family structure at $t=0\left(g_{\text {random }}=1\right)$ by using $N_{s o}=$ 10 sires and $N_{d o}=20$ dams. In schemes under truncation selection the numbers selected at $t>0$ were $N_{s}=10$ and $N_{d}=20$. The number of candidates across generations $(N)$ was 120 . The polygenic and the environmental variances were $\sigma_{u}^{2}=0.2$ and $\sigma_{e}^{2}=0.8$, respectively, giving a polygenic heritability of 0.2 . The effect of the QTL was completely additive with $a=0.5 \sigma_{p}$ (where $\sigma_{p}^{2}=\sigma_{u}^{2}+\sigma_{e}^{2}$ ). The initial frequency of the favourable allele was 0.15 . Thus at the founder generation $\left(t=-1\right.$ with $\left.g_{\text {random }}=1\right)$, the additive variance explained by the QTL and the total heritability were $\sigma_{v}^{2}=0.0638$ and $h_{t}^{2}=0.25$, respectively. 
Two flanking markers with six equifrequent alleles each were simulated. The distance between each marker and the QTL $(d)$ was $10 \mathrm{cM}$.

Alternative schemes considered different numbers of extra generations of random selection prior to selection $\left(g_{\text {random }}=4\right)$, different distances between each flanking marker and the QTL $(d=0.05,1,5,10,20$ and $30 \mathrm{cM})$ and different numbers of alleles for the markers (12 alleles of equal frequencies). Simulations with a large number of flanking markers (40) were also run. In schemes where prior information on the QTL effects was considered, it was assumed that this information was unbiased and obtained independently from another population. Different accuracies for the prior were considered and expressed as the number of "phantom" offspring ( $n$; see Appendix B). At any given round of selection, all current candidates (or only male candidates) were assumed to have prior information on the QTL. For a candidate $i$, its prior information $\hat{v}_{i}^{*}$ was assumed to be its true genotype effect regressed by the squared accuracy of the prior (i.e. $\left.\hat{v}_{i}^{*}=v_{i}^{*} \rho_{i}^{*}\right)$.

The number of replicates varied from five hundred to a thousand, depending on the method of selection (less replicates were run when selection was on the markers due to computing requirements).

\section{RESULTS}

The results presented are conditional on the survival of the favourable QTL allele (i.e. replicates where the allele was lost in any generation were excluded). However, for all the parameters and schemes studied, the probability of survival was always very close to one (i.e. higher than 0.99) except for the PHE schemes. In the latter, the survival rate was 0.985 and 0.989 for truncation and optimised selection, respectively. Given the small number of replicates where the favourable allele was lost, their exclusion from the analysis was not expected to introduce any significant bias in the results presented.

\subsection{Benefit from GAS and MAS with optimised and truncation selection}

Table I shows the total accumulated gain and the frequency of the favourable allele for the QTL over generations for the three types of basic schemes (GAS, MAS and PHE) under truncation and optimised selection. MAS was carried out assuming that the QTL was situated in the middle of a marker bracket of $20 \mathrm{cM}$ (i.e. the distance between each marker and the QTL was $10 \mathrm{cM})$. In order to make an objective comparison between both methods of selection, the rate of inbreeding used in the optimised scheme was restricted to the same value as that obtained with truncation selection $(\Delta F \approx 5 \%)$. The increase in inbreeding was maintained at the desired constant rate with optimised selection (results 
Table I. Total accumulated genetic gain $(G)$ and frequency of the favourable allele $(p)$ across generations $(t)$ obtained from truncation and optimised BLUP selection. Selection was on two flanking markers each $10 \mathrm{cM}$ apart from the QTL (MAS), directly on the QTL (GAS), or ignoring genotype information (PHE). The initial $p$ was 0.15 . With optimised selection, $\Delta F$ was restricted to $5 \%$. $^{\dagger}$

\begin{tabular}{|c|c|c|c|c|c|c|}
\hline \multirow[b]{2}{*}{$t$} & \multicolumn{2}{|c|}{ GAS } & \multicolumn{2}{|c|}{ MAS } & \multicolumn{2}{|c|}{ PHE } \\
\hline & $G$ & $p$ & $G$ & $p$ & $G$ & $p$ \\
\hline \multicolumn{7}{|c|}{ Truncation selection } \\
\hline 1 & 0.482 & 0.42 & 0.429 & 0.28 & 0.416 & 0.26 \\
\hline 2 & 0.957 & 0.76 & 0.819 & 0.45 & 0.774 & 0.39 \\
\hline 3 & 1.351 & 0.95 & 1.207 & 0.64 & 1.133 & 0.53 \\
\hline 4 & 1.633 & 0.99 & 1.561 & 0.80 & 1.474 & 0.67 \\
\hline 5 & 1.883 & 1.00 & 1.871 & 0.91 & 1.794 & 0.78 \\
\hline 6 & 2.116 & 1.00 & 2.137 & 0.96 & 2.079 & 0.86 \\
\hline 7 & 2.340 & 1.00 & 2.377 & 0.98 & 2.343 & 0.92 \\
\hline 8 & 2.558 & 1.00 & 2.591 & 0.99 & 2.580 & 0.95 \\
\hline 9 & 2.764 & 1.00 & 2.796 & 1.00 & 2.794 & 0.98 \\
\hline 10 & 2.959 & 1.00 & 2.987 & 1.00 & 2.997 & 0.99 \\
\hline \multicolumn{7}{|c|}{ Optimal selection } \\
\hline 1 & 0.568 & 0.50 & 0.468 & 0.29 & 0.460 & 0.28 \\
\hline 2 & 1.184 & 0.91 & 0.956 & 0.51 & 0.915 & 0.45 \\
\hline 3 & 1.573 & 0.99 & 1.439 & 0.75 & 1.361 & 0.62 \\
\hline 4 & 1.866 & 1.00 & 1.845 & 0.90 & 1.775 & 0.77 \\
\hline 5 & 2.152 & 1.00 & 2.174 & 0.97 & 2.144 & 0.87 \\
\hline 6 & 2.422 & 1.00 & 2.459 & 0.99 & 2.466 & 0.93 \\
\hline 7 & 2.685 & 1.00 & 2.715 & 1.00 & 2.754 & 0.97 \\
\hline 8 & 2.930 & 1.00 & 2.969 & 1.00 & 3.014 & 0.99 \\
\hline 9 & 3.167 & 1.00 & 3.206 & 1.00 & 3.260 & 0.99 \\
\hline 10 & 3.394 & 1.00 & 3.431 & 1.00 & 3.487 & 1.00 \\
\hline
\end{tabular}

$\dagger$ Standard errors ranged from 0.002 to 0.013 for total genetic values and from 0.0 to 0.01 for frequency of the favourable allele.

not shown) and consequently the accumulated inbreeding was very similar for the schemes compared. With optimised selection, the optimum number of individuals selected (which was practically constant after $t=1$ ) was the same for both sexes (around 9 males and 9 females) and for the three types of selection (GAS, MAS and PHE). These values were lower than the numbers selected under truncation selection (10 males and 20 females). 
The trend in genetic gain obtained with MAS schemes showed a similar pattern, in qualitative terms, to that observed with GAS (Tab. I, Figs. 1a and 1d). With both truncation and optimised selection, MAS produced extra gains in earlier generations relative to phenotypic selection (PHE) through a faster increase in the frequency of the favourable allele. Also, the lower rate in the polygenic gain observed with MAS relative to PHE in the early generations (see Figs. 1b and 1e) led to lower long-term gains in the MAS schemes.

The early benefit of using MAS was substantially smaller than the benefit from GAS. For the genetic parameters used in Table I, the extra gains of MAS relative to PHE were the highest at generations 3 (optimised selection) and 4 (truncation selection) and they were around 6\%. This value represented less than half the benefit achieved with GAS over PHE for these generations (11\% and $16 \%$ for truncation and optimised selection, respectively). The advantage of GAS over MAS was even higher at generations 2 (optimised selection) and 3 (truncation selection) where GAS had the maximum benefit over PHE. On the contrary, the loss in accumulated gain in the longer term obtained with GAS relative to PHE was much smaller when using MAS. By generation 9, the favourable allele was almost fixed in all truncation selection schemes $(p \geq 0.98)$ and the total genetic gain from MAS was still greater than that obtained with PHE. The greatest long-term loss relative to PHE was observed in optimised GAS schemes.

The optimised selection schemes followed the same pattern in gain from GAS and MAS relative to PHE as truncation selection schemes but yielded a greater benefit. Additionally, the optimisation of contributions also increased the relative advantage over PHE of including the information on the QTL via the genotype of the QTL itself. The peak of maximum gain was also achieved faster with optimisation than with truncation selection (see also Fig. 1). After the first generation of selection, the gain achieved when selecting on the markers was from 15 to $24 \%$ higher with an optimised selection than with a truncation selection. By generation 7, when the gene frequency was about 0.97 or higher, the genetic gain of the optimised PHE was greater than both GAS and MAS using truncation selection.

\subsection{Effect of recombination between the markers and the QTL}

Figure 1 shows the results of GAS compared to different MAS scenarios with varying distance $(d)$ between each of the two markers bracketing the QTL position and the QTL itself. The results shown are for optimised and truncation selection schemes and for total and polygenic gain expressed as a deviation from the gain achieved with the corresponding PHE scheme. Changes in the frequency of the favourable allele over generations are also shown. For all $d$ values, the general pattern was the same as that described above for $d=10 \mathrm{cM}$ (Tab. I). In general, GAS outperformed all MAS schemes in the 
Truncation
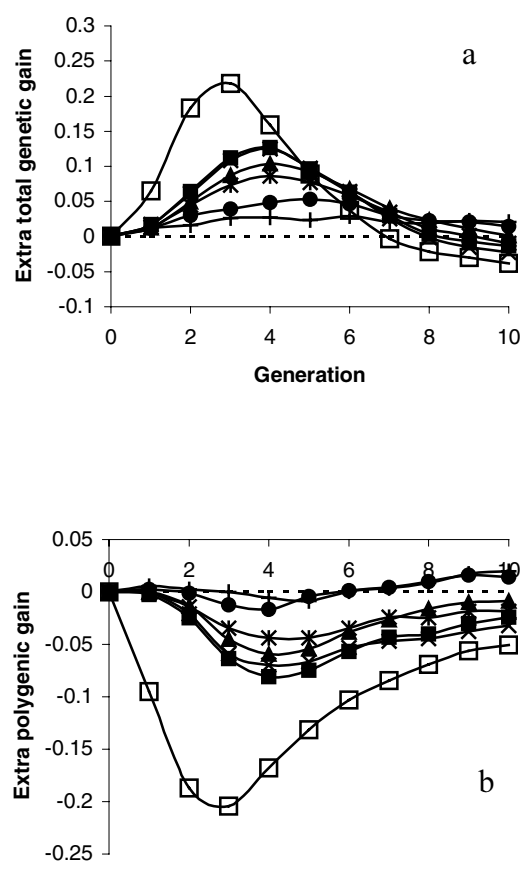

Generation

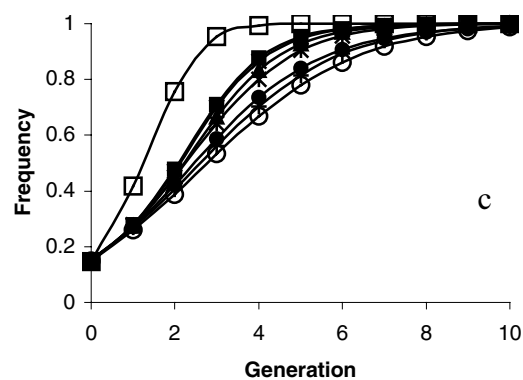

Optimised
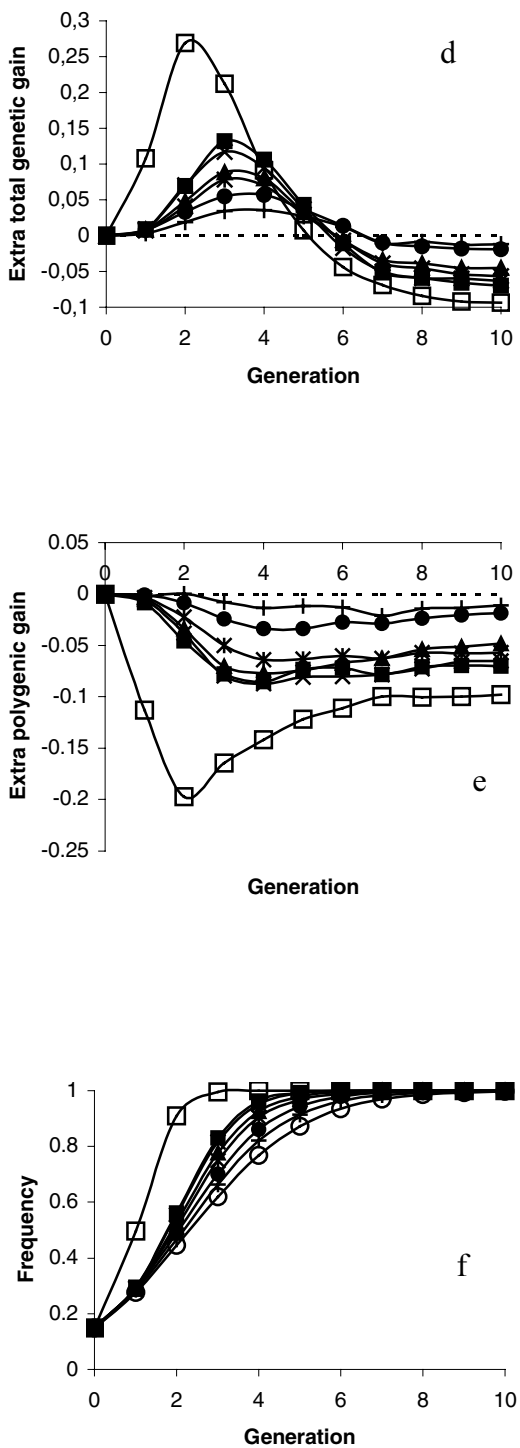

Figure 1. Accumulated total and polygenic genetic gains and frequency of the favourable allele over generations obtained from truncation and optimised BLUP selection on the QTL (GAS) and on two flanking markers (MAS) differing in the distance $(d)$ between each marker and the QTL. Results for genetic gains are expressed as deviations from gains from selection ignoring genotype information (PHE). $\square$ : GAS; $\mathbf{\square}$ : MAS, $d=0.05 ; \times$ : MAS, $d=1.0 ; \boldsymbol{\Delta}:$ MAS, $d=5.0 ; *:$ MAS, $d=10.0 ; \bullet:$ MAS, $d=20.0 ;+$ MAS, $d=30.0 ;$ o: PHE. 
early generations of selection, but MAS surpassed the performance of GAS in later generations, especially with the optimised schemes. The optimisation of contributions led to a faster increase in the frequency of the favourable allele (relative to truncation selection), particularly in GAS schemes and the early loss of polygenic gain in these schemes was high.

The narrower the marker bracket, the closer the response to selection in MAS schemes was to the response in GAS (Fig. 1). However, the results from MAS were somewhat disappointing in that, even with markers only $0.05 \mathrm{cM}$ away from the QTL position, MAS achieved only a small proportion of the extra gain obtained with GAS in the early generations. This low benefit of MAS was more accentuated in the first generation of selection where the extra gain from MAS relative to PHE was only around $20 \%$ of that achieved with GAS. Across all MAS schemes, the maximum accumulated benefit over PHE occurred between generations 3 and 4, representing, at most, half of the maximum benefit achieved by GAS (observed earlier, between generations 2 and 3).

Among the MAS schemes, those that had greater gains in early generations had lower gains in later generations. However, with truncation selection, some cases within the MAS schemes which achieved greater gain than PHE in early generations were not necessarily associated with a lower accumulated gain in later generations. In some scenarios $(e . g . d=10)$, MAS truncation selection schemes yielded a greater short-term gain than PHE but had no or little detrimental effects in the accumulated gain at generation 10 (Fig. 1a). At this generation, the favourable allele was practically fixed in all MAS schemes (Fig. 1c) and their cumulated total gain was still higher than with PHE in some cases. The long-term loss in genetic gain in MAS schemes was clearer with optimised selection (Fig. 1d).

For all values of $d$, the genetic gains achieved with the optimised schemes were higher than the gains achieved with truncation selection (results not shown except for $d=10 \mathrm{cM}$ in Tab. I). As mentioned above, optimised selection increased the relative advantage of GAS over PHE. However, the relative advantage of MAS schemes over PHE was similar for truncation and optimised selection.

\subsection{Effect of using prior information on the QTL effects}

Figure 2 shows gains obtained with truncation and optimised selection when prior information on the QTL was included in the mixed model equations. Two flanking markers each $10 \mathrm{cM}$ away from the QTL were simulated. Different accuracies for the prior estimate of the QTL effect were considered. These values, which refer only to the QTL, were $0.14,0.40,0.81$ and 0.98 and corresponded to $n=1, n=10, n=100$ and $n=1000$, respectively (see Appendix B). 


\section{Truncation}

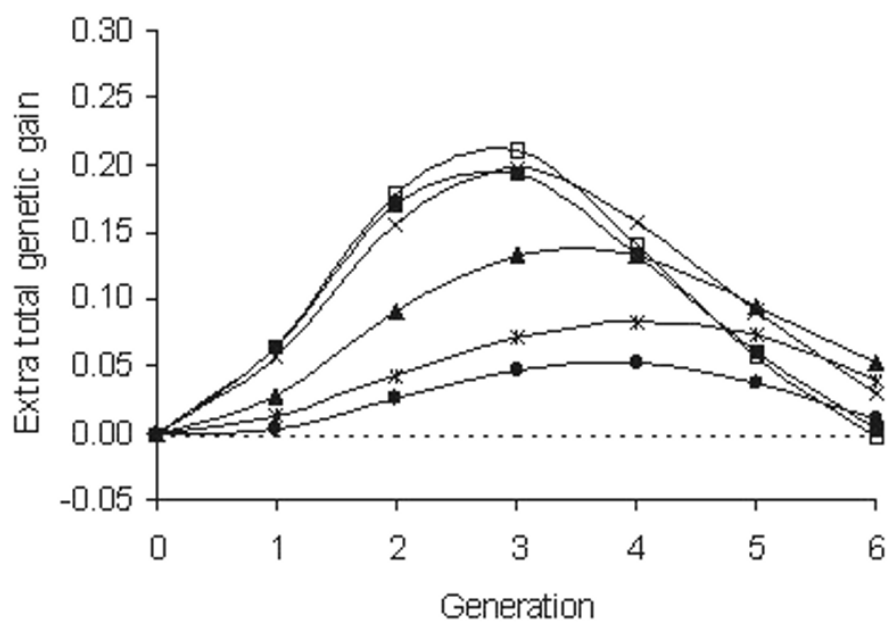

Optimised

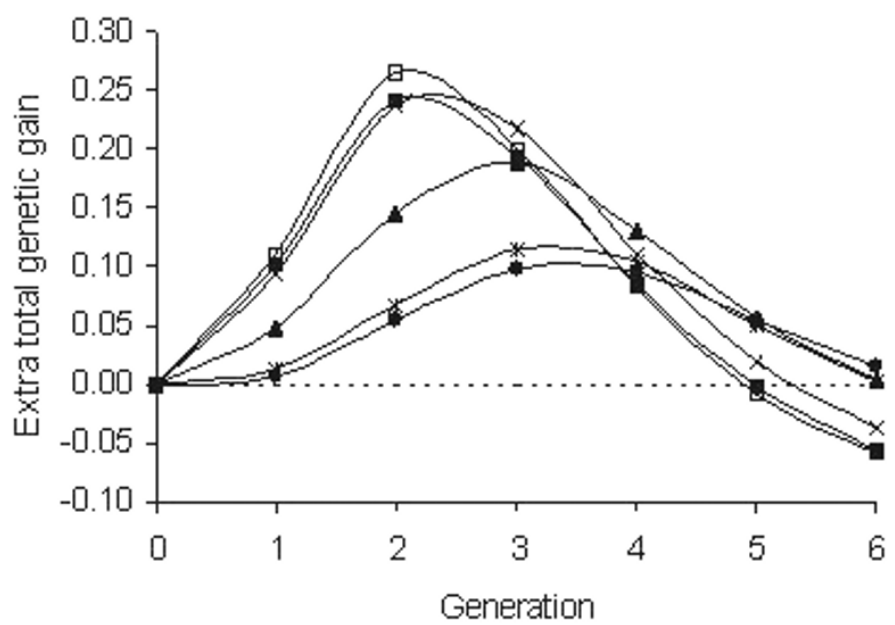

Figure 2. Total accumulated genetic gain over generations obtained from truncation and optimised BLUP selection on the QTL (GAS) and on two flanking markers with $(n>0)$ and without prior $(n=0)$ information. Here, $n$ is the number of "phantom" offspring. The results are expressed as deviations from gains from selection ignoring genotype information (PHE). $\square$ : GAS; $\mathbf{\square}$ : MAS, $n=1000 ; \times$ : MAS, $n=100$; $\boldsymbol{\Delta}$ : MAS, $n=10 ; *:$ MAS, $n=1 ; \bullet:$ MAS, $n=0$. 
The results indicate a continuous early response according to the amount of prior information (Fig. 2). This was due to an increase in the accuracy in predicting QTL effects by increasing the amount of information. With MAS and $\rho^{*}=0.81$, the response obtained was already very close to that obtained when selecting directly on the QTL (GAS) and very little improvement was observed when increasing $\rho^{*}$ from 0.81 to 0.98 . In other words, the accuracy $\rho^{*}=0.81$ was already sufficiently high to obtain accurate estimates. However, even when using priors of low accuracy $\left(\rho^{*}=0.14\right)$ there was a clear improvement in the response obtained compared to the response from standard MAS.

A situation more likely to be found in practice is presented in Figure 3. Here, only one sex (the males) had prior information. Also, records were only available for females. A comparison with the results described above indicates similar trends to those reported in previous studies for standard MAS without the use of priors (e.g. [27]). Although lower gains were obtained for the sex-limited trait than for the trait recorded in both sexes, MAS appeared to have more potential (relative to PHE) for the former type of trait. The use of prior information only for the males also substantially increased the potential of MAS.

\section{DISCUSSION}

This study investigated the benefits from marker assisted selection under clear and realistic assumptions (i.e. unambiguous model, phase of the markers unknown) when the genetic contributions of the candidates for selection are optimised for maximising the rate of genetic progress while restricting the rate of inbreeding to a specific value. Different schemes (i.e. GAS, MAS and PHE) were compared at the same rate of inbreeding. This represents an improvement over previous studies evaluating the benefit of MAS that have focussed on genetic gains obtained under truncation selection $[20,22,26$, 27 and that have assumed known marker haplotypes when estimating QTL effects $[26,27]$. Another novel aspect of this study was the inclusion of prior information on the QTL effects in the genetic evaluation of MAS schemes.

The optimisation of genetic contributions had a much bigger impact on genetic response than the use of markers. Significantly higher gains were obtained, in all cases, with optimised selection when compared to gains from truncation selection. The benefits from the optimised contributions were in line with those previously published. Villanueva et al. [31] have already shown that optimised selection ignoring all genotype information does as well as truncation GAS in the short-term and better in the long-term.

The optimisation method used here maximised genetic gain from the parental to the offspring generation while imposing a restriction on the rate of inbreeding. The emphasis given to the estimated breeding value (EBV) for 


\section{Truncation}

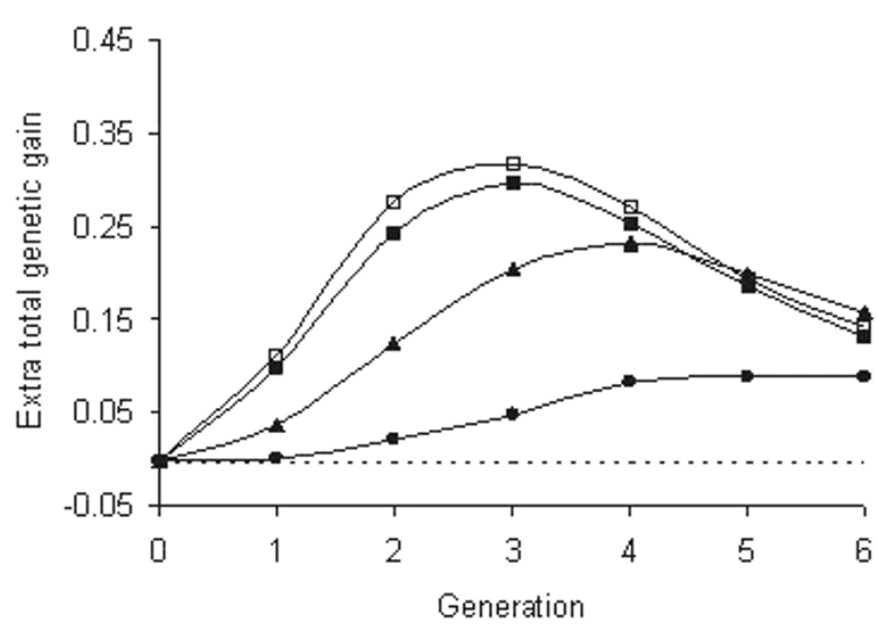

Optimised

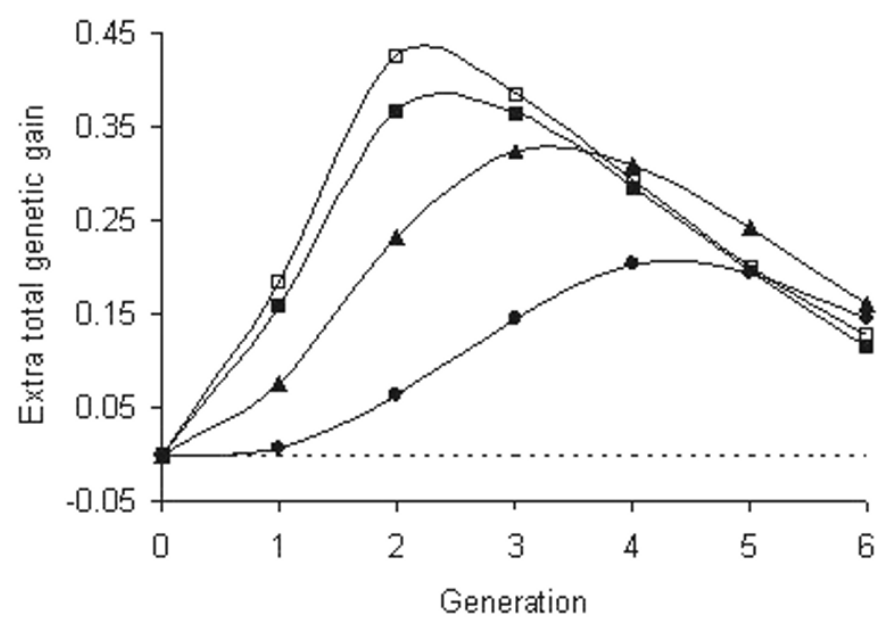

Figure 3. Total accumulated genetic gain over generations obtained from truncation and optimised BLUP selection on the QTL (GAS) and on two flanking markers with $(n>0)$ and without prior $(n=0)$ information. Here, $n$ is the number of "phantom" offspring. Selection was for a sex limited trait and prior information was only available for males. The results are expressed as deviations from gains from selection ignoring genotype information (PHE). $\square$ : GAS; $\mathbf{\square}$ : MAS, $n=1000 ; \boldsymbol{\Delta}$ : MAS, $n=10$; $\bullet:$ MAS, $n=0$. 
the QTL (relative to the polygenic EBV) in the selection criterion was fixed and therefore not optimal. This led to the previously described finding that the extra gains expected from GAS and MAS (relative to PHE) in the early generations of selection are not maintained in the long-term. The loss in longterm response of GAS and MAS was initially described for schemes under mass truncation selection (e.g. [11,24]). Villanueva et al. [31] showed that the conflict between long- and short-term responses from explicit use of the known gene could disappear in schemes with constrained inbreeding, and where the basis of evaluation is BLUP. However, this was only valid for scenarios where the gene had a larger effect than that considered here $(a=2.0$ versus $a=0.5)$. When the sum of genetic levels over generations $\left(G_{1}+\cdots+G_{10}\right)$ was considered, GAS produced the highest value and PHE produced the lowest but the differences between schemes were very small (results not shown).

Dekkers and van Arendonk [7] optimised the relative weight given to the QTL over generations and avoided the detrimental long-term effect. However, they assumed fixed contributions of candidates and no accumulation of inbreeding. The combined optimisation of contributions of selection candidates and weights on the QTL across generations could allow substantial increases in gain at a fixed rate of inbreeding and avoid the conflict between short- and long-term responses in GAS schemes [30].

The use of markers, in addition to optimised contributions, led to only moderate extra gains in the short term. The responses from MAS were intermediate to those obtained by selecting directly on the QTL and those obtained in conventional schemes that ignore molecular information. However, for the size of the population considered here, a substantial reduction in response was observed before fixation in both truncation and optimised selection when selecting on the markers rather than on the QTL itself, even with a recombination rate between the markers and the QTL as low as 0.0005 $(d=0.05)$. This value for $d$ might be unrealistic in practice but it was chosen to provide an indication of the potential upper limit of the genetic progress expected from MAS. The disadvantage of MAS relative to GAS in the short term was also observed for traits that can benefit more from MAS (i.e. lowly heritable and sex-limited traits). Also, the relatively low performance of MAS remained similar when the number of alleles per marker was increased from 6 to 12 , suggesting that the low performance of MAS was not due to a lack of information on the marker genotypes used during the selection process. Similarly, schemes with intermediate initial frequency of the favourable allele $(p=0.5)$, schemes with selection on a large number of markers (i.e. 40) and schemes with QTL effects normally distributed also showed this loss in gain when using MAS (results not shown).

In previous studies, the benefits from MAS have been found to be very variable depending on the genetic model assumed, the population structure and 
the time horizon [28]. Our truncation selection results are in line with those found by Ruane and Colleau [26] who assumed similar models and structures to those simulated here (i.e. mixed inheritance model, one single biallelic additive QTL flanked by two polymorphic markers, BLUP genetic evaluation model of Fernando and Grossman). Their results showed only a small short-term advantage of MAS over PHE (i.e. less than 4\%). A scheme under truncation selection using a set of their parameters $\left(d=10 \mathrm{cM}, p=0.5, \sigma_{u}^{2}=0.4375\right.$, $\sigma_{v}^{2}=0.625, \sigma_{e}^{2}=1.5, N_{s}=8, N_{d}=16$ and $N=128$ ) was simulated and produced similar results to those found by Ruane and Colleau [26]. Higher benefits from genotype information would be expected when that information is used at selection stages where limited or no phenotypic information is available to distinguish selection candidates.

Meuwissen and Goddard [20] found large benefits from MAS but their results are not comparable to those presented here for several reasons. Firstly, their use of the term "recombination rate" $(r)$ is not the standard one. Generally, recombination rate between two loci is defined as a function of their distance only, while they defined $r$ as "the probability that the Mendelian sampling of the QTL alleles could not be followed by the marker haplotypes... due to recombination within the marker haplotype but also due to markers being non-informative, or the haplotype not being known with certainty". Thus their term $r$, depends not only on the distance between the markers and the QTL loci but also on the "informativeness" of marker loci. This means that their $r$ is more a "traceability coefficient" rather than the recombination rate per se. They consider a range of values for $r$ from 0.05 to 0.4 which would correspond to values for the true recombination rate much lower than those considered here (e.g. given the marker allele frequency assumed in this study, a recombination rate 0.1 is equivalent to their " $r$ " being higher than 0.3 ). Secondly, combining together the effect of marker distance and marker information into a single parameter assumes that the informativeness of the markers remains constant over the selection process. This may prove to be an overoptimistic assumption since selection would change the frequency of the QTL producing a "hitch-hike" effect on the linked markers. Since some marker alleles may be lost, the information content of the linked markers may also decrease. The similar results obtained here for markers with 6 and 12 alleles suggest that the probability of losing alleles may be high. Finally, they did not allow double recombinations to occur except for one case (i.e. $r=0.4$; see their Tab. V). Double recombinations could play a role in determining the value of MAS but, given their definition of $r$, it is unclear what this role is. The assumptions made by Meuwissen and Goddard [20] may explain why their conclusions about the value of MAS were more optimistic than ours. We would argue that allowing for double recombinations and, especially, for the marker information to decay over the selection process are more realistic assumptions. 
The truncation selection schemes simulated by Meuwissen and Goddard [20] contained five ancestral generations with information on the markers available before the start of MAS. This extra information could have helped to have high accuracy in the estimation of the QTL effects and to obtain their large benefits from MAS, particularly in the first generations of selection. In our case, responses in the first generation of MAS were much closer to the responses obtained when ignoring genotypic information (PHE) than to the responses obtained from GAS. In order to investigate if the availability of more pedigree generations improve the accuracy (and therefore responses) four generations of random selection were simulated prior to generation zero (results not shown). The increased amount of marker genotype information at generation zero significantly increased the accuracy of the estimation of the QTL effects (from 0.54 to 0.65 ) but did not lead to higher gains. Also, when the assumption of a biallelic QTL was relaxed by simulating normally-distributed allelic effects, as in [20], the responses from MAS were still substantially lower than those from GAS. Thus, the higher benefits from MAS observed by Meuwissen and Goddard [20] could be due to the unrealistic assumptions implied in their study that have been mentioned above.

The disappointing results of MAS when compared to GAS were due to two facts. Firstly, with MAS, selection on the QTL is indirect (as it is applied on the markers) rather than direct as with GAS. Secondly, with MAS, the QTL effects are estimated from the data rather than being known, as with GAS. Schemes where genotypes of the individuals were known but QTL effects need to be estimated would reduce the advantage of GAS. However, if the population size were large enough we may assume that the QTL effects would be well estimated. The fact that even with a very close marker bracket the early benefits of MAS were far from those with GAS shows the importance of knowing the genotypes for the QTL (i.e. cloning the QTL) once it has been mapped.

The attractiveness of the MAS evaluation method proposed by Fernando and Grossman [9] is its versatile use under different situations by carrying out the evaluation under a BLUP framework. The QTL information is summarised and included in the mixed model as the variance explained by the QTL and its position. The QTL position and the marker genotypes are used to calculate the IBD matrix needed in the evaluation. The variance explained by the QTL combines information on the QTL effect and its gene frequency but no knowledge on the magnitudes of these two parameters is considered by the mixed model. The results comparing gains obtained selecting directly on the QTL and responses selecting on the markers show that the basic mixed model approach of Fernando and Grossman [9] includes a restricted amount of information about the QTL which may explain the reduced benefit from MAS relative to GAS. 
The results presented in this study showed that including prior information about the QTL effects of the candidates for selection substantially improves the response to selection. The magnitude of the extra response increased according to the accuracy of the extra information. The improvement in the response was to the extent that selection on the markers using very accurate prior information ( $\rho^{*}>0.80$ through a modified version of the Fernando and Grossman method) could be as good as when selecting directly on the QTL. Surprisingly, even with the lowest accuracy considered ( $\rho^{*}=0.14$ for $\left.n=1\right)$, the increase in response was significant. This may partly be due to the fact that the prior information of an individual was assumed to be the true genotype effect (regressed by the squared accuracy of the prior) rather than being sampled from a distribution. Thus the results on the benefit of including priors into the evaluation described here may be overoptimistic but they clearly show the potential of using prior information on the QTL effect into the MAS evaluation.

Hence, given that there is scope for improvement by adding extra information on the QTL, it is important to determine the type of information available, assess the methodology for including such information and quantify the magnitude of the improvement when doing so. The prior information needs to be independent of the information available from the population under selection (marker genotypes and performance records). The methodology for adding prior information on the QTL effects that has been presented here may require modification if other types of prior are going to be used.

Therefore, further challenges in the process of incorporating MAS into practical breeding programmes should include the (i) identification of additional information which can be obtained for the mapped QTL to be used in a specific breeding scheme; and (ii) adaptation of MAS methods to include this information. The type and amount of extra information on the mapped QTL will vary accordingly with the breeding schemes. They may include knowledge of the gene frequency, genotype probability for the candidates for selection, population linkage disequilibrium between the markers and the QTL or a combination of these. For instance, QTL mapping using the granddaughter design commonly used in dairy cattle populations would also identify heterozygous individuals and the average allele substitution. QTL mapping studies in other animal species have been successful in estimating the effect of the QTL $[5,6,32]$. Because of the wide variety of the extra information available, the ways of including this into the evaluation procedure would also expect to differ accordingly. Methodology to include knowledge on the population linkage disequilibrium between the markers and the QTL has already been proposed [21].

The simple rules derived by Henderson [14] and Quaas [25] to obtain the inverse of the A matrix made the application of BLUP animal models to large data sets possible. In the same way, the application of BLUP animal models 
including marker information in practical breeding programmes will depend, in most livestock species, on the development of efficient algorithms to obtain the inverse of the IBD matrix. These developments and the possible use of available extra information on the QTL could broaden the use of MAS for improving selection responses.

\section{ACKNOWLEDGEMENTS}

This work was funded by the Biotechnology and Biological Sciences Research Council (BBSRC), the Pig Improvement Company (PIC), GenusHolland Genetics Joint Venture and the Meat and Livestock Commission (MLC) through the LINK Sustainable Livestock Production Programme. SAC also receives financial support from the Scottish Executive Environment and Rural Affairs Department (SEERAD). Work in the Roslin Institute receives support from the Department for Environment, Food and Rural Affairs (DEFRA). We thank Prof. G. Simm for useful comments on the manuscript.

\section{REFERENCES}

[1] Ashwell M.S., Van Tassell C.P., Detection of putative loci affecting milk, health, and type traits in a US Holstein population using 70 microsatellite markers in a genome scan, J. Dairy Sci. 82 (1999) 2497-2502.

[2] Avendaño S., Villanueva B., Woolliams J.A., Expected increases in genetic merit in the UK Aberdeen Angus beef cattle breed from applying optimised selection, in: Proceedings of the British Society of Animal Science, 8-10 April 2002, University of York, York, p. 54.

[3] Avendaño S., Villanueva B., Woolliams J.A., Optimisation of selection decisions in the UK Meatlinc breed of sheep, in: Proceedings of the British Society of Animal Science, 8-10 April 2002, University of York, York, p. 194.

[4] Cantet R.J.C., Smith C., Reduced animal model for marker assisted selection using best linear unbiased prediction, Genet. Sel. Evol. 23 (1991) 221-233.

[5] Casas E., Stone R.T., Keele J.W., Shackelford S.D., Kappes S.M., Koohmaraie M., A comprehensive search for quantitative trait loci affecting growth and carcass composition of cattle segregating alternative forms of the myostatin gene, J. Anim. Sci. 79 (2001) 854-860.

[6] Cassady J.P., Johnson R.K., Pomp D., Rohrer G.A., Van Vleck L.D., Spiegel E.K., Gilson K.M., Identification of quantitative trait loci affecting reproduction in pigs, J. Anim. Sci. 79 (2001) 623-633.

[7] Dekkers J.C.M., van Arendonk J.A.M., Optimizing selection for quantitative traits with information on an identified locus in outbred populations, Genet. Res., Camb. 71 (1998) 257-275.

[8] Falconer D.S., Mackay T.F.C., Introduction to quantitative genetics, 4th edn., Longman, 1996. 
[9] Fernando R.L., Grossman M., Marker assisted selection using best linear unbiased prediction, Genet. Sel. Evol. 21 (1989) 467-477.

[10] Fournet F., Elsen J.M., Barbieri M.E., Manfredi E., Effect of including major gene information in mass selection: a stochastic simulation in a small population, Genet. Sel. Evol. 29 (1997) 35-56.

[11] Gibson J.P., Short term gain at the expense of long term response with selection on identified loci, in: Proceedings of the 5th World Congress on Genetics Applied to Livestock Production, 7-12 August 1994, Vol. 21, University of Guelph, Guelph, pp. 201-204.

[12] Goddard M.E., A mixed model for analyses of data on multiple genetic markers, Theor. Appl. Genet. 83 (1992) 878-886.

[13] Grundy B., Villanueva B., Woolliams J.A., Dynamic selection procedures for constrained inbreeding and their consequences for pedigree development, Genet. Res., Camb. 72 (1998) 159-168.

[14] Henderson C.R., A simple method for computing the inverse of a numerator relationship matrix used in prediction of breeding values, Biometrics 32 (1976) 69-83.

[15] Hoeschele I., Elimination of quantitative trait loci equations in an animal model incorporating genetic marker data, J. Dairy Sci. 76 (1993) 1693-1713.

[16] Ikonen T., Bovenhuis H., Ojala M., Ruottinen O., Georges M., Associations between casein haplotypes and first lactation milk production traits in Finnish Ayrshire cows, J. Dairy Sci. 84 (2001) 507-514.

[17] Larzul C., Manfredi E., Elsen J.M., Potential gain from including major gene information in breeding value estimation, Genet. Sel. Evol. 29 (1997) 161-184.

[18] Lynch M., Walsh B., Genetics and analysis of quantitative traits, 1st edn., Sinauer Associates, Sunderland, 1998.

[19] Meuwissen T.H.E., Maximizing the response of selection with a predefined rate of inbreeding, J. Anim. Sci. 75 (1997) 934-940.

[20] Meuwissen T.H.E., Goddard M.E., The use of marker haplotypes in animal breeding schemes, Genet. Sel. Evol. 28 (1996) 161-176.

[21] Meuwissen T.H.E., Hayes B.J., Goddard M.E., Prediction of genetic value using genome-wide dense marker maps, Genetics 157 (2001) 1819-1829.

[22] Meuwissen T.H.E., van Arendonk J.A.M., Potential improvements in rate of genetic gain from marker-assisted selection in dairy cattle breeding schemes, J. Dairy Sci. 75 (1992) 1651-1659.

[23] Pong-Wong R., George A.W., Woolliams J.A., Haley C.S., A simple and rapid method for calculating identity-by-descent matrices using multiple markers, Genet. Sel. Evol. 33 (2001) 453-471.

[24] Pong-Wong R., Woolliams J.A., Response to mass selection when an identified major gene is segregating, Genet. Sel. Evol. 30 (1998) 313-337.

[25] Quaas R.L., Computing the diagonal elements and inverse of a large numerator relationship matrix, Biometrics 32 (1976) 949-953.

[26] Ruane J., Colleau J.J., Marker assisted selection for genetic improvement of animal populations when a single QTL is marked, Genet. Res., Camb. 66 (1995) 71-83.

[27] Ruane J., Colleau J.J., Marker-assisted selection for a sex-limited character in a nucleus breeding population, J. Dairy Sci. 79 (1996) 1666-1678. 
[28] Spelman R.J., Major factors in marker-assisted selection genetic response in dairy cattle populations, in: Proceedings of the 6th World Congress on Genetics Applied to Livestock Production, 11-16 January 1998, Vol. 26, University of New England, Armidale, pp. 365-368.

[29] Van Arendonk J.A.M., Tier B., Kinghorn B.P., Use of multiple genetic markers in prediction of breeding values, Genetics 137 (1994) 319-329.

[30] Villanueva B., Dekkers J.C.M., Woolliams J.A., Settar P., Maximising genetic gain with QTL information and control of inbreeding, in: Proceedings of the 7th World Congress on Genetics Applied to Livestock Production, CD-ROM communication no. 22-18.

[31] Villanueva B., Pong-Wong R., Grundy B., Woolliams J.A., Potential benefit from using an identified major gene and BLUP estimated breeding values in selection programmes, Genet. Sel. Evol. 31 (1999) 115-133.

[32] Walling G.A, Visscher P.M., Andersson L., Rothschild M.F., Wang L., Moser G., Groenen M.A.M., Bidanel J.-P., Cepica S., Archibald A.L., Geldermann H., de Koning D.J., Milan D., Haley C.S., Combined analyses of data from quantitative trait loci mapping studies: Chromosome 4 effects on porcine growth and fatness, Genetics 155 (2000) 1369-1378.

[33] Wang T., Fernando R.L., VanderBeek S., Grossman M., Van Arendonk J.A.M., Covariance between relatives for a marked quantitative trait locus, Genet. Sel. Evol. 27 (1995) 251-272.

\section{APPENDIX A}

\section{Inclusion of prior information on the QTL effects in the MA-BLUP evaluation}

Let us assume that additional to the marker genotype information, some candidates also have independent prior information about the QTL effect. Thus, for an individual $i, \hat{v}_{i}^{*}$ is an estimate (with a certain accuracy $\rho_{i}^{*}$ ) of the combined additive effects of its two QTL alleles.

Hence, the objective is to combine into the evaluation, both the prior information and the data of the population with appropriate weighting factors. In order to achieve that, the QTL estimates $\left(\hat{v}_{i}^{*}\right)$ and their accuracies $\left(\rho_{i}^{*}\right)$ were transformed into a number of half-sib "phantom" offspring of $i$, each with one phenotypic record. The transformed data can, then, be included into a BLUP as suggested by Fernando and Grossman [9] and, therefore, making it possible to be combined together with the data of the selected population into a single evaluation procedure. The calculation of the number of offspring and their phenotype from $\hat{v}_{i}^{*}$ and $\rho_{i}^{*}$ for individual $i$, is shown in Appendix B.

Since $\hat{v}_{i}^{*}$ contains information only on the QTL effect, the statistical model for the phenotypes of the "phantom" offspring is:

$$
y_{o(i)}^{*}=(0.5) \mu+(0.5) \mu^{*}+v_{o(i)}^{i}+v_{o(i)}^{x}+e_{o(i)}
$$


where $y_{o(i)}^{*}$ is the phenotypic value of one "phantom" offspring of individual $i$, $\mu$ the overall mean of the current population under selection, $\mu^{*}$ is the overall mean of the population from which the prior information came from, and $v_{o(i)}^{i}$ and $v_{o(i)}^{x}$ are the effects of the QTL alleles of the offspring inherited from $i$ and a "phantom" mate of $i$, respectively.

Then, in order to account for the prior information in the evaluation, the BLUP of Fernando and Grossman [9] was extended to include some extra parameters. The mixed model equations (MME) given in the Methods section were augmented to include the extra mean $\left(\mu^{*}\right)$, the effects of the two alleles of the "phantom" offspring $\left(v_{o(i)}^{i}\right.$ and $\left.v_{o(i)}^{x}\right)$ and the effects of "phantom" mate alleles $\left(v_{x(i)}^{p}\right.$ and $\left.v_{x(i)}^{m}\right)$. Since the prior information is an estimate of the QTL effect, the equations related to the polygenic effects in the mixed model were not affected. Since the estimated allele effects for each "phantom" offspring and for the mate are not needed in the selection decisions, all $n_{i}$ "phantom" offspring of individual $i$ can be added together in a single equation (i.e. estimating a combined effect of the "phantom" offspring QTL effect). Hence, assuming that $h$ individuals have prior information, the MME would need to be augmented to include $4 h+1$ extra parameters.

\section{Left hand side of the MME}

Let $\mathbf{C}$ be the left hand side of the MME augmented with the extra $4 h+1$ parameters. Let $\mu^{*}, v_{o(i)}^{i}, v_{o(i)}^{x}, v_{x(i)}^{p}$ and $v_{x(i)}^{m}$ be the index denoting the extra rows and columns added in $\mathbf{C}$ to account for the prior mean, the effect of the alleles of the "phantom" offspring inherited from $i$ and mate $x$, and the effects of the paternal and maternal alleles of the "phantom" mate of $i$, respectively. Also, let $\mu$ be the index for the position of the population mean and $v_{i}^{p}$ and $v_{i}^{m}$ be the index denoting the positions for the paternal and maternal QTL effects of the individual $i$.

The process for constructing the matrix $\mathbf{C}$ would be to start filling it with the terms arising from the data of the evaluated population (see Methods section) and, after that, filling it with the other terms related to the records of the "phantom" offspring. For the latter, the $4 h+1$ extra rows and columns are initially set to zero. Then, for each individual $i$ with prior information:

(1) add $(0.25) n_{i}$ to the positions $\mathbf{C}[\mu, \mu], \mathbf{C}\left[\mu^{*}, \mu\right], \mathbf{C}\left[\mu, \mu^{*}\right], \mathbf{C}\left[\mu^{*}, \mu^{*}\right]$;

(2) add $(0.50) n_{i}$ to the positions $\mathbf{C}\left[\mu, v_{o(i)}^{i}\right], \mathbf{C}\left[\mu^{*}, v_{o(i)}^{i}\right], \mathbf{C}\left[v_{o(i)}^{i}, \mu^{*}\right]$, $\mathbf{C}\left[v_{o(i)}^{i}, \mu^{*}\right]$

(3) add (0.50) $n_{i}$ to the positions $\mathbf{C}\left[\mu, v_{o(i)}^{x}\right], \mathbf{C}\left[\mu^{*}, v_{o(i)}^{x}\right], \mathbf{C}\left[v_{o(i)}^{x}, \mu^{*}\right]$, $\mathbf{C}\left[v_{o(i)}^{x}, \mu^{*}\right]$

(4) add $n_{i}$ to the positions $\mathbf{C}\left[v_{o(i)}^{i}, v_{o(i)}^{i}\right], \mathbf{C}\left[v_{o(i)}^{x}, v_{o(i)}^{x}\right], \mathbf{C}\left[v_{o(i)}^{i}, v_{o(i)}^{x}\right]$, $\mathbf{C}\left[v_{o(i)}^{x}, v_{o(i)}^{i}\right]$. 
The matrix $\mathbf{C}$ also needs to be modified to account for the extra terms in the $\mathbf{G}$ matrix arising from adding the "phantom" offspring. Assuming that the marker genotypes of the "phantom" offspring are non-informative, their IBD values in the $\mathbf{G}$ matrix depend only on the pedigree information. Therefore, the inverse of $\mathbf{G}$ can be updated using similar rules as those suggested by Henderson [13] for the $\mathbf{A}$ matrix. Thus $\mathbf{C}$ needs to be further modified as follow:

For the terms involving $v_{o(i)}^{i}$,

(1) add $n_{i}\left[(0.5) /\left(1-f_{i}\right)\right] \gamma_{2}$ to the positions $\mathbf{C}\left[v_{i}^{p}, v_{i}^{p}\right], \mathbf{C}\left[v_{i}^{m}, v_{i}^{m}\right], \mathbf{C}\left[v_{i}^{p}, v_{i}^{m}\right]$, and $\mathbf{C}\left[v_{i}^{m}, v_{i}^{p}\right]$

(2) add $n_{i}\left[-1 /\left(1-f_{i}\right)\right] \gamma_{2}$ to the positions $\mathbf{C}\left[v_{i}^{p}, v_{o(i)}^{i}\right], \mathbf{C}\left[v_{o(i)}^{i}, v_{i}^{p}\right], \mathbf{C}\left[v_{i}^{m}, v_{o(i)}^{i}\right]$ and $\mathbf{C}\left[v_{o(i)}^{i}, v_{i}^{m}\right]$

(3) add $n_{i}\left[2 /\left(1-f_{i}\right)\right] \gamma_{2}$ to the position $\mathbf{C}\left[v_{o(i)}^{i}, v_{o(i)}^{i}\right]$;

where $f_{i}$ is the IBD value between the two gametes of $i\left(v_{i}^{p}\right.$ and $\left.v_{i}^{m}\right)$ and $\gamma_{2}$ is the variance ratio $\sigma_{e}^{2} /(0.5) \sigma_{v}^{2}$.

For the terms involving $v_{o(i)}^{x}$, the IBD between the two gametes of the mate of $i$, $\left(\right.$ i.e. $\left.f_{x}\right)$ is assumed to be zero. Then:

(1) add (0.5) $n_{i} \gamma_{2}$ to the positions $\mathbf{C}\left[v_{x(i)}^{p}, v_{x(i)}^{p}\right], \mathbf{C}\left[v_{x(i)}^{m}, v_{x(i)}^{m}\right], \mathbf{C}\left[v_{x(i)}^{p}, v_{x(i)}^{m}\right]$ and $\mathbf{C}\left[v_{x(i)}^{m}, v_{x(i)}^{p}\right]$

(2) add $-n_{i} \gamma_{2}$ to the positions $\mathbf{C}\left[v_{x(i)}^{p}, v_{o(i)}^{x}\right], \mathbf{C}\left[v_{o(i)}^{x}, v_{x(i)}^{p}\right], \mathbf{C}\left[v_{x(i)}^{m}, v_{o(i)}^{x}\right]$ and $\mathbf{C}\left[v_{o(i)}^{x}, v_{x(i)}^{m}\right]$

(3) add $3 n_{i} \gamma_{2}$ to the position $\mathbf{C}\left[v_{o(i)}^{x}, v_{o(i)}^{x}\right]$.

\section{Right hand side of the MME}

As with $\mathbf{C}$, the right hand side vector (RHS) of the augmented MME is, first, filled with the terms resulting from the data (see Methods section). For the inclusion of the "phantom" offspring's records, the $4 h+1$ extra rows are initially set to zero. Then, following the same notation for the indices denoting rows and columns, the right hand side (RHS) of the MME is modified as follows. For each individual $i$ with prior information:

(1) add $(0.5) n_{i} y_{o(i)}^{*}$ to the positions $\mathbf{R H S}[\mu]$ and $\mathbf{R H S}\left[\mu^{*}\right]$;

(2) add $n_{i} y_{o(i)}^{*}$ to the positions $\mathbf{R H S}\left[v_{o(i)}^{i}\right]$ and $\mathbf{R H S}\left[v_{o(i)}^{x}\right]$.

\section{APPENDIX B}

\section{Computing the expected offspring phenotype and the number} of offspring from the prior estimate of the QTL effect and its accuracy

Assume that the records available to predict the QTL breeding value of individual $i$ are the average performance of its $n_{i}$ offspring $\left(\bar{y}_{o(i)}\right)$. The total phenotypic value of the one individual "phantom" offspring of $i$ is:

$$
y_{o(i)}^{*}=(0.5) \mu+(0.5) \mu^{*}+v_{o(i)}^{i}+v_{o(i)}^{m}+e_{o(i)}
$$


where $\mu$ is the overall mean of the current population under selection, $\mu^{*}$ is the mean of the population from where the prior information came from, and $v_{o(i)}^{i}$ and $v_{o(i)}^{m}$ are the effects of the alleles inherited from $i$ and a "phantom" mate of $i$, respectively and $e_{o(i)}$ is the residual effect. Now let $h_{v}^{2}$ be $\sigma_{v}^{2} / \sigma_{p}^{2}$ and $\sigma_{p}^{2}$ be $\sigma_{v}^{2}+\sigma_{e}^{2}$.

The prior estimate of the QTL breeding value of individual $i$ is:

$$
\hat{v}_{i}^{*}=b_{i} \bar{y}_{o(i)}^{*}
$$

where $\hat{v}_{i}^{*}=\hat{v}_{i}^{p *}+\hat{v}_{i}^{m *}$ and $b_{i}$ is the weight obtained from the standard index selection theory,

$$
b_{i}=\operatorname{Cov}\left(v_{i}, \bar{y}_{o(i)}^{*}\right) / \operatorname{Var}\left(\bar{y}_{o(i)}^{*}\right) .
$$

Assuming that QTL and environmental effects are uncorrelated,

$$
\begin{aligned}
\operatorname{Cov}\left(v_{i}, \bar{y}_{o(i)}^{*}\right) & =(1 / 2) \sigma_{v}^{2} \\
\operatorname{Var}\left(\bar{y}_{o(i)}^{*}\right) & =\left(1 / n_{i}\right) \sigma_{p}^{2}+\left[\left(n_{i}-1\right) / n_{i}\right](1 / 4) \sigma_{v}^{2}
\end{aligned}
$$

where $n_{i}$ is the number of offspring. Then,

$$
b_{i}=\frac{2 n_{i} h_{v}^{2}}{4+\left(n_{i}-1\right) h_{v}^{2}} .
$$

The accuracy of the estimate $\hat{v}_{i}^{*}$ is

$$
\rho_{i}^{*}=\operatorname{Cov}\left(v_{i}, \hat{v}_{i}^{*}\right) / \sqrt{\operatorname{Var}\left(v_{i}\right) \operatorname{Var}\left(\hat{v}_{i}^{*}\right)}
$$

which reduces to $\rho_{i}^{*}=\sqrt{(1 / 2) b_{i}}$ since $\operatorname{Cov}\left(v_{i}, \hat{v}_{i}^{*}\right)=\operatorname{Var}\left(\hat{v}_{i}^{*}\right)$.

The number of "phantom" offspring $\left(n_{i}\right)$ can be derived by substituting the expression for $b_{i}$ into the expression for $\rho_{i}^{*}$ and solving for $n_{i}$,

$$
n_{i}=\frac{\rho_{i}^{* 2}\left(h_{v}^{2}-4\right)}{h_{v}^{2}\left(\rho_{i}^{* 2}-1\right)} .
$$

Similarly, the average phenotypic value of the "phantom" offspring $\left(\bar{y}_{o(i)}^{*}\right)$ can be expressed as a function of the accuracy and the prior estimate of the QTL effect:

$$
\bar{y}_{o(i)}^{*}=\hat{v}_{i}^{*} \frac{1}{2 \rho_{i}^{* 2}} .
$$

